\title{
THERMODYNAMICS OF THE HARMONIC OSCILLATOR: WIEN'S DISPLACEMENT LAW AND THE PLANCK SPECTRUM
}

\author{
Timothy H. Boyer \\ Department of Physics, City College of the City \\ University of New York, New York, NY 10031
}

\begin{abstract}
A thermodynamic analysis of the harmonic oscillator is presented. Motivation for the study is provided by the blackbody radiation spectrum; when blackbody radiation is regarded as a system of noninteracting harmonic oscillator modes, the thermodynamics follows from that of the harmonic oscillators. Using the behavior of a harmonic oscillator thermodynamic system under an adiabatic change of oscillator frequency $\omega$, we show that the thermodynamic functions can all be derived from a single function of $\omega / T$, analogous to Wien's displacement theorem. The high- and low-frequency energy limits allow asymptotic energy forms involving $T$ alone or $\omega$ alone, corresponding to energy equipartition and zero-point energy. It is suggested that the "smoothest possible" function which behaves as $a-b x$ at small values of $x$ and vanishes at large $x$ is $a e^{-b x / a}$ because it is a monotonic analytic function every derivative of which is a multiple of the function itself. In this sense, it is noted that the Planck spectrum with zero-point radiation corresponds to the function satisfying the Wien displacement result which provides the smoothest possible interpolation between energy equipartition at low frequency and zero-point energy at high frequency.
\end{abstract}




\section{INTRODUCTION}

Blackbody radiation holds an unusual place in the physics curriculum. Blackbody radiation provides a simple system which can be used as a model for thermodynamic analysis showing the derivation of the Stefan-Boltzmann law connecting total radiation energy and temperature[1]. Also, thermal radiation can be regarded as a collection of harmonic radiation modes[2] which can be treated by Doppler shift from a moving piston to obtain the Wien displacement law relating to the blackbody spectrum[3]. However, the full blackbody spectrum is regarded as inaccessible from thermodynamics; rather the blackbody spectrum reappears in statistical mechanics where it is used as an illustration of the breakdown of classical statistical mechanics and the need for energy quanta[4].

In this article we depart from the traditional points of view. We treat blackbody radiation by analyzing the thermodynamics of the harmonic oscillator. Although the harmonic oscillator is often discussed in statistical mechanics, it is not usually analyzed in thermodynamics[5]. We begin by noting the expression for the work done by a harmonic oscillator on slow change of its frequency. Then we use the laws of thermodynamics to give a simple but unfamiliar derivation of Wien's displacement law[6]. This law is thus seen to hold not only for electromagnetic radiation but also for any harmonic oscillator which is a thermodynamic system at temperature $T$. Next we discuss all the thermodynamic functions associated with a harmonic oscillator thermodynamic system. We note that the high- and low-temperature limits allowed by the Wien law correspond to zero-point energy and energy equipartition. This simple observation is again not traditional in the physics literature. Finally, we suggest a natural interpolation between the high- and low-temperature forms allowed by the Wien displacement law. The simple interpolation leads to the Planck spectrum with zero-point energy.

The unconventional treatment presented here provides a compact approach to the blackbody problem through an elementary thermodynamic system which is simple enough for the textbooks. The analysis emphasizes the intimate connections between blackbody radiation and zero-point radiation, and also fits neatly with the modern realization that constant acceleration of a harmonic oscillator through zero-point radiation makes the oscillator energy equal to the Planck spectrum with zero-point radiation[7].

\section{THERMODYNAMICS OF A HARMONIC OSCILLATOR}

A harmonic oscillator corresponds to a nonrelativistic point mass $m$ moving in one dimension in a potential $V=(1 / 2) m \omega^{2} x^{2}$. It can be described by the Lagrangian $L(x, \dot{x})=$ $(1 / 2) m \dot{x}^{2}-(1 / 2) m \omega^{2} x^{2}$, and corresponds to harmonic oscillation $x=(2 \mathcal{U} / m \omega)^{1 / 2} \cos (\omega t+\phi)$ at energy $\mathcal{U}$ and angular frequency $\omega$. Under a slow change in the spring constant of the system corresponding to a change in the natural angular frequency $\omega$, the energy divided by the frequency $\mathcal{U} / \omega$ is a constant[8] and so the change in system energy is $d \mathcal{U}=(\mathcal{U} / \omega) d \omega$ and the work $d W$ done by the system on the external agency changing the frequency is

$$
d W=-(\mathcal{U} / \omega) d \omega .
$$

Thus we regard $\mathcal{X}=-\mathcal{U} / \omega$ as the generalized force associated with a change in frequency $\omega$.

If a harmonic oscillator is weakly coupled to a heat bath at temperature $T$, then it will exchange energy with the heat bath. In equilibrium with the bath, the harmonic oscillator at 
frequency $\omega$ will have an average energy $\mathcal{U}(\omega \cdot T)$. Thus the oscillator forms a thermodynamic system suitable for treatment by the usual methods of thermodynamics involving (average) energy $\mathcal{U}$, parameter $\omega$, and associated work $d W=-(\mathcal{U} / \omega) d \omega$.

\section{DERIVATION OF FUNCTIONAL DEPENDENCE CORRESPONDING TO WIEN'S DISPLACEMENT THEOREM}

When the oscillator is at thermal equilibrium in a bath at temperature $T$, the oscillator will have an (average) energy $\mathcal{U}(\omega, T)$ and an entropy $\mathcal{S}(\omega, T)$ depending upon the temperature $T$ and natural oscillation frequency $\omega$. Using the work expression in(1), the laws of thermodynamics for the oscillator give

$$
d Q=T d \mathcal{S}(T, \omega)=d \mathcal{U}(T, \omega)-(\mathcal{U} / \omega) d \omega
$$

This can be rewritten as

$$
T\left[\left(\frac{\partial \mathcal{S}}{\partial T}\right)_{\omega} d T+\left(\frac{\partial \mathcal{S}}{\partial \omega}\right)_{T} d \omega\right]=\left(\frac{\partial \mathcal{U}}{\partial T}\right)_{\omega} d T+\left(\frac{\partial \mathcal{U}}{\partial \omega}\right)_{T} d \omega-\frac{\mathcal{U}}{\omega} d \omega
$$

Since the variables $\omega$ and $T$ are independent, this requires

$$
T\left(\frac{\partial \mathcal{S}}{\partial T}\right)_{\omega}=\left(\frac{\partial \mathcal{U}}{\partial T}\right)_{\omega}, \quad T\left(\frac{\partial \mathcal{S}}{\partial \omega}\right)_{T}=\left(\frac{\partial \mathcal{U}}{\partial \omega}\right)_{T}-\frac{\mathcal{U}}{\omega}
$$

Differentiating the first equation of (4) with respect to $\omega$ and the second with respect to $T$ gives

$$
T \frac{\partial^{2} \mathcal{S}}{\partial \omega \partial T}=\frac{\partial^{2} \mathcal{U}}{\partial \omega \partial T}, \quad\left(\frac{\partial \mathcal{S}}{\partial \omega}\right)_{T}+T \frac{\partial^{2} \mathcal{S}}{\partial T \partial \omega}=\frac{\partial^{2} \mathcal{U}}{\partial T \partial \omega}-\frac{1}{\omega}\left(\frac{\partial \mathcal{U}}{\partial T}\right)_{\omega}
$$

Subtracting the first equation of (5) from the second as as to eliminate the second-derivative terms, we have

$$
\left(\frac{\partial \mathcal{S}}{\partial \omega}\right)_{T}=-\frac{1}{\omega}\left(\frac{\partial \mathcal{U}}{\partial T}\right)_{\omega}
$$

Next using (6) together with the first equation of (4), we find

$$
\left(\frac{\partial \mathcal{S}}{\partial \omega}\right)_{T}=-\frac{T}{\omega}\left(\frac{\partial \mathcal{S}}{\partial T}\right)_{\omega}
$$

which has the general solution

$$
\mathcal{S}(\omega, T)=g(\omega / T)
$$

where $g$ is an arbitrary function of the single variable $\omega / T$. On the other hand if we use the second equation of (4), then we find from Eq.(6)

$$
\left(\frac{\partial \mathcal{U}}{\partial \omega}\right)_{T}-\frac{\mathcal{U}}{\omega}=-\frac{T}{\omega}\left(\frac{\partial \mathcal{U}}{\partial T}\right)_{\omega}
$$

which has the general solution

$$
\mathcal{U}(\omega, T)=\omega f(\omega / T)
$$

where $f$ is an arbitrary function of the single variable $\omega / T$. This corresponds to the information in the Wien displacement theorem. [3] Although the Wien theorem is often derived from the doppler shift of electromagnetic waves reflected from a moving piston in a cavity, our analysis shows that it holds in thermal equilibrium for any system described by a simple harmonic oscillator Lagrangian. 


\section{THERMODYNAMIC FUNCTIONS FOR A HARMONIC OSCILLATOR}

The Wien displacement results $\mathcal{S}(\omega, T)=g(\omega / T)$ and $\mathcal{U}(\omega, T)=\omega f(\omega / T)$ give constraints upon all the thermodynamic functions for a harmonic oscillator. The thermodynamic potential[9] $\phi(\omega / T)$, from which all the other thermodynamics functions may be derived, must be a function of the combination $\omega / T$. The average oscillator energy $\mathcal{U}$ in thermal equilibrium follows as

$$
\mathcal{U}(\omega, T)=T^{2}\left(\frac{\partial \phi}{\partial T}\right)_{\omega}=-\omega \phi^{\prime}(\omega / T)
$$

The entropy $\mathcal{S}$ of the oscillator is again a function of $\omega / T$,

$$
\mathcal{S}(\omega / T)=\phi(\omega / T)+\mathcal{U}(\omega, T) / T=\phi(\omega / T)-(\omega / T) \phi^{\prime}(\omega / T)
$$

The Helmholtz free energy $\mathcal{F}$ is directly related to the thermodynamic potential $\phi(\omega / T)$

$$
\mathcal{F}(\omega, T)=-T \phi(\omega / T)
$$

The generalized force $\mathcal{X}$ associated with a change in $\omega$ is

$$
\mathcal{X}(\omega / T)=T\left(\frac{\partial \phi}{\partial \omega}\right)_{T}=\phi^{\prime}(\omega / T)
$$

and the specific heat $\mathcal{C}$ at constant $\omega$ is given by

$$
\mathcal{C}(\omega / T)=\left(\frac{\partial \mathcal{U}}{\partial T}\right)_{\omega}=\left(\frac{\omega}{T}\right)^{2} \phi^{\prime \prime}(\omega / T)
$$

Thus the equilibrium thermodynamics of a classical harmonic oscillator system is determined by one function, the unknown function $\phi(\omega / T)$.

\section{WIEN DISPLACEMENT RESULT AND ZERO-POINT ENERGY}

There are two natural extremes for the oscillator energy given by the Wien displacement result in (11); one extreme makes the energy $\mathcal{U}(\omega, T)$ independent of temperature $T$, and the other makes the energy $\mathcal{U}(\omega, T)$ independent of the natural frequency $\omega$.

We deal first with the temperature-independent energy. When the potential function $\phi^{\prime}(\omega / T)=-$ const and so

$$
\phi(\omega / T)=- \text { const } \times(\omega / T)
$$

then the oscillator energy in (11) takes the form

$$
\mathcal{U}(\omega, T)=\mathcal{U}_{z p}(\omega)=\text { const } \times \omega
$$

This corresponds to temperature-independent zero-point energy.

We note that substitution of the zero-point energy (17) into the first law of thermodynamics in the form

$$
d Q=d \mathcal{U}-(\mathcal{U} / \omega) d \omega=d(\text { const } \times \omega)-(\text { const } \times \omega / \omega) d \omega=0
$$


tells us that no heat $d Q$ enters the system on changing the natural frequency of the oscillator $\omega$. Thus changes in zero-point energy occur without any change in the thermodynamic entropy $\mathcal{S}(\omega / T)$ of the system. Indeed, we see that if $\phi_{z p}^{\prime}$ is constant, then $\phi_{z p}(\omega / T)=$ const $\times \omega / T$ must be linear in its argument, and the entropy $\mathcal{S}$ in Eq.(12) vanishes for any function $\phi$ which is linear in its argument.

Zero-point energy is random energy which is present even at zero temperature. Thermodynamics allows the possibility of zero-point energy and experimental evidence (such as that for van der Waals forces) requires its existence.[10] It is natural to choose the unknown constant for the zero-point energy in (17) so as to fit the experimentally measured van der Waals forces. This corresponds to an oscillator energy

$$
\mathcal{U}_{z p}(\omega)=(1 / 2) \hbar \omega
$$

where $\hbar$ is a constant which takes the value familiar for Planck's constant.

\section{WIEN DISPLACEMENT RESULT AND ENERGY EQUIPARTITION}

The other extreme for the Wien displacement result (11) is the case where the oscillator energy depends upon the temperature but has no dependence upon the natural oscillator frequency $\omega$. Thus when $\phi^{\prime}(\omega / T)=-$ const $/(\omega / T)$ in equation (11), then the oscillator energy is

$$
\mathcal{U}(\omega, T)=\mathcal{U}_{R J}(T)=\text { const } \times T .
$$

This is the familiar energy equipartition law (proposed by Rayleigh and Jeans for lowfrequency radiation modes) where the constant is chosen as Boltzmann's constant $k_{B}$,

$$
\mathcal{U}_{R J}(T)=k_{B} T \text {. }
$$

In this case, an isothermal change of the natural oscillator frequency $\omega$ produces no change in the oscillator internal energy. Rather, from (2), the isothermal work done on changing the natural frequency $\omega$ is provided by heat added which keeps the internal oscillator energy constant,

$$
d Q=T d \mathcal{S}(\omega / T)=d \mathcal{U}_{R J}(T)-(\mathcal{U} / \omega) d \omega, \quad \text { constant } T
$$

Then

$$
d \mathcal{S}_{R J}=0-\left(k_{B} / \omega\right) d \omega, \quad \text { constant } T,
$$

and since we know the functional form $\mathcal{S}(\omega / T)$, we have the familiar result

$$
\mathcal{S}_{R J}(\omega / T)=-k_{B} \ln (\omega / T)+\text { const }
$$

Indeed if $\phi_{R J}^{\prime}(\omega / T)=-k_{B} /(\omega / T)$, then $\phi_{R J}(\omega / T)=-k_{B} \ln (\omega / T)$ and the entropy in (12) takes the form (24).

\section{USE OF NATURAL UNITS IN THE ANALYSIS}

In this paper we are not interested in the numerical evaluation of thermodynamic quantities but rather in the fundamental thermodynamic behavior. On this account we will measure all quantities in terms of energy and take the entropy as a pure number. Thus we 
will take $\hbar=1$ and measure frequencies in energy units. Also, we will take $k_{B}=1$ and measure temperature in energy units.[11] Thus the limiting form corresponding to zero-point energy has

$$
\phi_{z p}(\omega / T)=-(1 / 2)(\omega / T), \quad \mathcal{U}_{z p}(\omega)=(1 / 2) \omega
$$

while the limiting form corresponding to energy equipartition becomes

$$
\phi_{R J}(\omega / T)=-\ln (\omega / T), \quad \mathcal{U}_{R J}(T)=T
$$

\section{ASYMPTOTIC LIMITS FOR THERMAL OSCILLATOR ENERGY}

In general, the behavior of an oscillator system will depend upon both frequency $\omega$ and temperature $T$ as in (11). In the limit as $T \rightarrow 0$, we expect to recover the zero-point energy of the oscillator

$$
\lim _{T \rightarrow 0} \mathcal{U}(\omega, T)=\lim _{T \rightarrow 0}\left[-\omega \phi^{\prime}(\omega / T)\right]=\mathcal{U}_{z p}(\omega)=(1 / 2) \omega
$$

and the associated thermodynamic potential

$$
\phi(\omega / T) \rightarrow \phi_{z p}(\omega / T)=-(1 / 2)(\omega / T)
$$

In the limit $\omega \rightarrow 0$, we expect to obtain the equipartition energy

$$
\lim _{\omega \rightarrow 0} \mathcal{U}(\omega, T)=\lim _{\omega \rightarrow 0}\left[-\omega \phi^{\prime}(\omega / T)\right]=\mathcal{U}_{R J}(T)=T
$$

and the associated thermodynamic potential

$$
\phi(\omega / T) \rightarrow \phi_{R J}(\omega / T)=-\ln (\omega / T)
$$

It is sometimes useful to make a distinction between the THERMAL energy $\mathcal{U}_{T}(\omega, T)$ of an oscillator and the oscillator's TOTAL energy $\mathcal{U}(\omega, T)$. The thermal energy is just the (average) energy above the (average) zero-point energy

$$
\mathcal{U}_{T}(\omega, T)=\mathcal{U}(\omega, T)-\mathcal{U}_{z p}(\omega)=-\omega \phi^{\prime}(\omega / T)-(1 / 2) \omega=-\omega\left[\phi^{\prime}(\omega / T)-\phi_{z p}^{\prime}(\omega / T)\right]
$$

Although the total oscillator energy $\mathcal{U}(\omega, T)$ is related to forces, it is only the thermal oscillator energy $\mathcal{U}_{T}(\omega, T)$ which is related to changes in thermodynamic entropy since (as seen above) $\phi_{z p}(\omega / T)$ does not give any thermodynamic entropy.

\section{PLANCK SPECTRUM AS THE SMOOTHEST INTERPOLATION BE- TWEEN THE EQUIPARTITION AND ZERO-POINT LIMITS}

Although the thermodynamic forms given in Eqs.(11)-(15) represent the information obtained from thermodynamic analysis, it is tempting to try to guess the full thermodynamic behavior which is chosen by nature. Now experiments indicate that nature indeed chooses the asymptotic forms given in Eqs.(27)-(30) corresponding to non-zero values of $\hbar$ and $k_{B}$. Thus we ask whether there is any natural choice for behavior which connects these asymptotic forms. One expects thermodynamics to involve smooth functions, and hence one might seek the "smoothest possible" interpolation between the extremes. 
Is the idea of a "smoothest possible" interpolation well-defined? In general, this seems ambiguous. However, there are cases where the "smoothest possible" function appears absolutely clear. Thus if for small values of $x$ an analytic function has the form $f(x)=$ $a-b x+O\left(x^{2}\right)$ with $a>0, b>0$, and for large values of $x$ the function $f(x) \rightarrow 0$, then the smoothest possible interpolation between the limits is $f(x)=a \exp (-b x / a)$. This function meets the asymptotic limits and is a monotonic function whose $n$th derivative is just $(-b / a)^{n}$ times the value of the function at that point. Thus every point $x$ involves the same local functional behavior for $f(x)$. Every point is equivalent. It is only modification of this functional form which introduces preferred values for $x$.

Let us reconsider the asymptotic forms given in Eqs.(27)-(30). The energy forms are not useful since these do not give us asymptotic functions of $\omega / T$. The limiting thermodynamic potentials

$$
\phi_{R J}(\omega / T)=-\ln (\omega / T), \quad \phi_{z p}(\omega / T)=-(1 / 2) \omega / T
$$

involve logarithmic behavior at the low-frequency limit. Since the logarithmic function is more complicated analytically than the exponential function, it is convenient to take the exponential of the negative of these functions and to consider

$$
\exp \left[-\phi_{R J}(\omega / T)\right]=\omega / T, \quad \exp \left[-\phi_{z p}(\omega / T)\right]=\exp [\omega /(2 T)]
$$

The exponentiation will not change the "smoothest possible" criterion required of the interpolation. Thus according to (33) we are searching for the "smoothest possible" interpolation $\exp [-\phi(\omega / T)]$ between linear behavior $\omega / T$ at small argument and exponential behavior $\exp [\omega /(2 T)]$ at large argument

$$
\exp [-\phi(\omega / T)] \rightarrow \omega / T \text { for } \omega / T \rightarrow 0, \text { and } \exp [-\phi(\omega / T)] \rightarrow \exp [\omega /(2 T)] \text { for } \omega / T \rightarrow \infty
$$

We notice that the difference of the high-frequency limit and the desired interpolation has the asymptotic forms

$$
\begin{gathered}
\exp [\omega /(2 T)]-\exp [-\phi(\omega / T)] \rightarrow 1+\omega /(2 T)-\omega / T=1-\omega /(2 T) \quad \text { for } \omega / T \rightarrow 0 \\
\exp [\omega /(2 T)]-\exp [-\phi(\omega / T)] \rightarrow 0 \text { for } \omega / T \rightarrow \infty
\end{gathered}
$$

But this corresponds to exactly the case mentioned in the previous paragraph. Thus the "smoothest possible" interpolation is

$$
\exp [\omega /(2 T)]-\exp [-\phi(\omega / T)]=\exp [-\omega /(2 T)]
$$

This implies

$$
\exp [-\phi(\omega / T)]=\exp [\omega /(2 T)]-\exp [-\omega /(2 T)]=2 \sinh [\omega /(2 T)]
$$

It is easy to see that the right-hand side of (37) has exactly the asymptotic forms demanded in (33). Then taking the logarithm of (37) and using the thermodynamic relations given in (11) and (12), this smooth interpolation leads to the thermodynamic functions

$$
\phi_{P z p}\left(\frac{\omega}{T}\right)=-\ln \left[2 \sinh \left(\frac{1}{2} \frac{\omega}{T}\right)\right]
$$




$$
\begin{gathered}
\mathcal{U}_{P z p}(\omega, T)=\frac{1}{2} \omega \operatorname{coth}\left(\frac{1}{2} \frac{\omega}{T}\right)=\frac{\omega}{\exp (\omega / T)-1}+\frac{1}{2} \omega \\
\mathcal{S}_{P}\left(\frac{\omega}{T}\right)=-\ln \left[2 \sinh \left(\frac{1}{2} \frac{\omega}{T}\right)\right]+\frac{1}{2} \frac{\omega}{T} \operatorname{coth}\left(\frac{1}{2} \frac{\omega}{T}\right)
\end{gathered}
$$

We have labeled these thermodynamic functions with the subscript "Pzp" or "P" because they correspond exactly to the familiar Planck average oscillator energy including zero-point energy. As noted above, the entropy depends upon the Planck thermal spectrum $\mathcal{U}_{T}(\omega, T)$ but does not reflect the zero-point energy included in $\mathcal{U}(\omega, T)$.

\section{COMMENTS ON THE INTERPOLATION FOR PLANCK'S SPECTRUM}

The analysis above shows that demanding the smoothest interpolation between the equipartition and zero-point limits suggests the Planck spectrum. This seems a surprisingly simple extrapolation from the thermodynamic analysis. One may wonder why such an extrapolation was not made a century ago. Indeed, many physicists are aware that Planck did arrive at the blackbody spectrum as an interpolation. However, Planck's interpolation did not come from the limits on the Wien displacement theorem. Rather, his interpolation involved a modification which combined earlier guesses at the high- and low-frequency parts of the thermal energy spectrum $\mathcal{U}_{T}(\omega, T)$.[12]

The simple interpolation made here was not made a century ago because physicists did not think in terms of a temperature-independent zero-point radiation. Indeed, even today, textbook discussions of blackbody radiation do not usually make any reference to zeropoint radiation.[13] It is only much more recently, and in particular in connection with experimental measurements of Casimir forces, that physicists have taken seriously the zeropoint energy suggested by the high-frequency limit of the Wien displacement law.

Indeed the smooth extrapolation analysis for a harmonic oscillator given here fits neatly with the thermal effects of acceleration suggested by Davies and Unruh[7]. If one considers a charged harmonic oscillator undergoing constant acceleration through zero-point radiation, then the oscillator comes into equilibrium with the random radiation. When the oscillator has zero acceleration, its average energy $\mathcal{U}(\omega, a=0)$ depends only upon its frequency and is just zero-point energy $\mathcal{U}(\omega, a=0)=(1 / 2) \hbar \omega$. When the oscillator has a large acceleration $a$, then the average oscillator energy depends only upon its acceleration $a$ and is independent of its natural frequency $\omega$. Thus zero-point energy and energy equipartition form the natural extremes. And the function which connects these extremes is found to be exactly the Planck spectrum (39) with a temperature $T=\hbar a / 2 \pi c k_{B} \cdot[7]$

\section{CONCLUDING SUMMARY}

The harmonic oscillator provides a simple thermodynamics system involving energy $\mathcal{U}$, temperature $T$, and oscillator frequency $\omega$. If one carries out a quasi-static change in the oscillator frequency $\omega$, then thermodynamic analysis leads to results which correspond to the Wien displacement law. All of the thermodynamic behavior for the oscillator can be derived from a single thermodynamic potential function $\phi(\omega / T)$ depending upon the single variable $\omega / T$. If we consider the limits which make the oscillator energy $\mathcal{U}(\omega, T)$ 
independent of one of its variables, then we find the extremes corresponding to zero-point energy and energy equipartition. Finally, as a guess for nature's choice of thermodynamic behavior, we ask for the function satisfying the Wien displacement law which provides the smoothest interpolation between the extremes of zero-point energy and energy equipartition. This leads to the Planck spectrum with zero-point radiation.

[1] See, for example, M. Planck, The Theory of Heat Radiation (Dover, New York 1959), pp. 61-63, or R. Becker and G. Leibfried, Theory of Heat 2nd ed. (Springer, New York 1967), pp. 16-17, or P.M. Morse, Thermal Physics 2nd ed (Benjamin/Cummings, Reading, MA 1969), pp. 78-79.

[2] See, for example, E. A. Power, Introductory Quantum Electrodynamics (American Elsevier, New York 1964), pp. 18-22.

[3] See, for example, M. Planck in reference 1, pp. 72-83, or F. K. Richtmyer, E. H. Kennard, and T. Lauritsen, Introduction to Modern Physics (McGraw-Hill, New York 1955), pp. 113-118, or B. H. Lavenda, Statistical Physics: a Probabilistic Approach (Wiley, New York 1991), pp. 67-69.

[4] See, for example, R. Reif, Fundamentals of Statistical and Thermal Physics (McGraw-Hill, New York 1965), pp. 55-56, 251-253, or R. Eisberg and R. Resnick, Quantum Physics of Atoms, Molecules, Solids, Nuclei, and Particles 2nd ed. (Wiley, New York 1985), pp. 6-19.

[5] I do not know of any text where the harmonic oscillator is treated as a thermodynamics system apart from statistical mechanics.

[6] I am not aware of any thermodynamic treatment of the adiabatic change of the harmonic oscillator frequency. The familiar derivations of the Wien law take the Doppler-shift form given in references 3 .

[7] P. C. W. Davies, "Scalar particle production in Schwarzschild and Rindler metrics," J. Phys. A 8, 609 (1975); W. G. Unruh, "Notes on black-hole evaporation," Phys. Rev. D 14, 871 (1976); T. H. Boyer, "Thermal effects of acceleration for a classical dipole oscillator in classical electromagnetic zero-point radiation," Phys. Rev. D 29, 1089-1095 (1984); D. C. Cole, "Properties of a classical charged harmonic oscillator accelerated through classical electromagnetic zero-point radiation," Phys. Rev. D 31, 1972-1981 (1985).

[8] The adiabatic invariance of $\mathcal{U} / \omega$ can be obtained by action-angle variables. See, for example, H. Goldstein, Classical Mechanics 2nd ed (Addison-Wesley, Reading, MA, 1980), p. 462. The situation involving adiabatic change in frequency is most famous in the case of a pendulum length which is changed slowly. See, for example, Goldstein's exercise 10, p. 543. An elementary derivation is given, for example, by T. H. Boyer, "Connection between the adiabatic hypothesis of old quantum theory and classical electrodynamics with classical electromagnetic zero-point radiation," Phys. Rev. A 18, 1238-1245 (1978), Eqs.(1)-(9).

[9] C. Garrod, Statistical Mechanics and Thermodynamics (Oxford, New York 1995), p. 128.

[10] M. J. Sparnaay, "Measurement of the attractive forces between flat plates," Physica 24, 751764 (1958); S. K. Lamoreaux, "Demonstration of the Casimir force in the 0.6 to $6 \mu \mathrm{m}$ range," Phys. Rev. Lett. 78, 5-8 (1997), 81, 5475-5476 (1998); U. Mohideen, "Precision measurement of the Casimir force from 0.1 to $0.9 \mu \mathrm{m}$," Phys. Rev. Lett. 81, 4549-4552 (1998); and H. B. Chan, V. A. Aksyuk, R. N. Kleiman, D. J. Bishop, and F. Capasso, "Quantum mechanical actuation of microelectomechanical systems by the Casimir force," Science 291, 1941-1944 
(2001).

[11] See the discussion of natural units by C. Garrod, reference 9, p. 120 . The choice $\hbar=1$ is familiar to particle physicists. The measurement of temperature in energy units is familiar in thermodynamics where our choice corresponds to the use of what is usually termed $\tau$ instead of T. See also, for example, C. Kittel, Elementary Statistical Physics (Wiley, New York 1958), p. 27.

[12] See, for example, the discussion by B. H. Lavenda in reference 3, pp. 74-76. See also, T. H. Kuhn, Black-Body Theory and the Quantum Discontinuity 1894-1912 (Oxford U. Press, New York 1978).

[13] I am not aware of any textbook treatment of blackbody radiation which mentions zero-point energy as part of the spectrum involved. 